\title{
Einfluss von Tempus und Aspekt auf die Wahl der Verbformen in schriftlichen Texten russischsprachiger Deutschlerner
}

The Influence of Tense and Aspect on the Choice of Verb Forms in Texts Written by Russian-speaking Learners of German

\section{Irina Mikhaylina}

Humboldt-Universität zu Berlin, Deutschland

This article investigates the influence of tense and aspect on the choice of verb forms in texts written by Russian-speaking learners of German. Through eight written narrations, each produced by advanced learners of German with L1-Russian and German native speakers, the use of verb forms and relevant linguistic means (perfect markers, temporal adverbs and temporal clauses) was compared and analysed. The study shows that even very advanced Russian-speaking learners of German could not meet target language preferences in German. They tended to deploy a different temporal perspective that German native speaker (simple past instead of present tense) and they also showed an overuse of the perfect tense, especially when describing completed actions. These differences compared to the preferences of German native speakers can be explained as transfer effects from the L1 of Russian-speaking learners since - unlike in German - the grammatical aspect in Russian is obligatory and its perfective form offers an effective tool to express completeness.

\section{$1 \quad$ Einleitung}

Nach langer Zeit der Zurückhaltung in der Linguistik bezüglich des Zusammenhangs sprachspezifischer Strukturen und kognitiver Prozesse gewinnt diese Frage mittlerweile an Interesse in der einschlägigen Forschung. Die Ergebnisse der aktuellen empirischen Studien im Bereich der Informationsstruktur und der Ereignis-Enkodierung (Caroll, Lambert 2003, Stutterheim 2003, Stutterheim, Caroll 2005, Schmiedtová, Sahonenko 2008) zeigen, dass es selbst fortgeschrittenen Lernenden schwer fällt, zielsprachliche Präferenzen in der Fremdsprache oder Zweitsprache (im Folgenden L2 genannt) zu erkennen. Diese Untersuchungen zum Zweitspracherwerb liefern Evidenz 
dafür, dass der Verwendung obligatorischer grammatischer Kategorien, wie z.B. des Aspekts in einigen Sprachen, ,highly automatized preferences“ (Schmiedtová, Stutterheim, Carroll, 2010: 66) zugrunde liegen, welche die Sprachproduktion bereits auf der Konzeptualisierungsebene bestimmen und dadurch die Wahl der sprachlichen Mittel in der L2 beeinflussen. Stutterheim, Carroll (2005:1) sprechen in diesem Zusammenhang vom „kognitiven Akzent“, der sich ,in der Wahl der sprachlichen Mittel und letztlich der Gestalt des Textes“" (ebd.) niederschlägt.

Laut Stutterheim (1986) basieren konzeptorientierte Studien im Bereich der Temporalität auf der Annahme, dass ,die besondere Ausprägung der Zeitkonzepte, über die ein erwachsener Sprecher verfügt, im Wesentlichen im Verlauf des Erstspracherwerbs festgelegt wird“"(Stutterheim, 1986:1).

Deutsch und Russisch weisen im Bereich der Temporalität maßgebliche Differenzen auf. Einer der größten Unterschiede besteht darin, dass die grammatische Kategorie des Aspekts im Russischen, anders als beim Deutschen, obligatorisch ist. Aspektuelle Konzepte sind aber universell und werden im Deutschen mit anderen sprachlichen Mitteln, überwiegend lexikalischen, ausgedrückt. In ihrer Studie zur Rolle des grammatischen Aspekts in der EreignisEnkodierung bringen Schmiedtová, Sahonenko (2008) empirische Evidenzen dafür, dass L2-Lerner ${ }^{1}$, die ,von einem reichen Tempus- und Aspektsystem kommen“ (ebd.: 54), im Deutschen trotzdem nach sprachlichen Ausdrucksmöglichkeiten für diese Kategorie suchen.

Die vorliegende Studie setzt sich zum Ziel, die möglichen Transfereffekte des russischen Aspekt- und Tempussystems auf die Wahl der sprachlichen Mittel zum Ausdruck der Temporalität im Deutschen zusammenzufassen und zu analysieren. Der Fokus wird dabei auf die schriftliche Textproduktion der fortgeschrittenen russischsprachigen Deutschlerner gelegt. Henning (2000: 4) führt aus, dass ,[g]eschriebene Sprache [...] stärker den traditionellen Normen des Tempusgebrauchs [folgt], während in der gesprochenen Sprache die Regeln bezüglich der Wahl eines Tempus - besonders zur Bezeichnung von Vergangenheit - nicht so strikt sind.“

\subsection{Theoretische Grundlagen}

Für die vorliegende Studie wird die Tempusdefinition von Comrie (1985: 9) angenommen: „tense is grammaticalised expression of location in time“. Temporale Relationen versteht Klein (1994: 65) als eine Art Verhältnis zwischen der Zeitspanne, die bereits gegeben wurde (Thema), und der später eingeführten

1 In der Studie von Schmiedtová, Sahonenko (2008) wurden die Daten von russischen und tschechischen Deutschlerner analysiert. 
Zeitspanne (Relatum). Demzufolge unterscheidet Klein (2009: 46) zwischen folgenden Bezugszeitspannen:

- Die Sprechzeit (time of utterance, TU. auch: Äußerungszeit) ist die Zeit, in der der Satz unmittelbar geäußert wird.

- Die Situationszeit (time of situation, TSit) ist die Zeit, in der die Situation ausgeführt wird.

- Die Topikzeit (topic time, TT) ist die Zeit bzw. die Zeitspanne, über die etwas geäußert wird.

Klein (2009: 24) sieht das Tempus nicht als eine Relation zwischen der Sprechzeit und der Situationszeit, sondern als eine Relation zwischen der Sprechzeit und der Topikzeit. Den Aspekt sieht Klein (1994: 24) als eine Relation zwischen der Topikzeit und der Situationszeit und definiert ihn als ,a particular viewpoint on the situation which is described by the sentence".

An dieser Stelle ist es nötig, kurz auf den Tempusansatz von Weinrich (1964, 2001) einzugehen. Nach Weinrich (2001: 32ff.) gehören die Tempora Präsens, Perfekt und beiden Futurformen zu der „besprechenden“ TempusGruppe-I. Die „erzählende“ Tempus-Gruppe-II beinhaltet Präteritum, Plusquamperfekt, Konditional und Konditional II. Anhand der Analyse literarischer Texte kommt Weinrich zu der Schlussfolgerung, dass sich in einigen literarischen Gattungen oder Sprechsituationen „die ziemlich eindeutige Dominanz entweder der Tempus-Gruppe-I oder der Tempus-Gruppe-II“ bestätigt.

Diese Einteilung erklärt Weinrich anhand der Differenz zwischen gespannter und entspannter Sprechhaltung. Die Funktion der erzählenden Tempora sieht Weinrich (2001: 51) darin, „dem Hörer einer Mitteilung die Nachricht davon zu geben, dass diese Mitteilung ,nur' eine Erzählung ist, so dass der Hörer mit einer gewissen Gelassenheit zuhören kann“, zudem in einer Besprechsituation der Hörer die Dinge ,im Modus der Betroffenheit aufnehmen soll" (Weinrich, 2001: 50). Die Tempora in beiden Gruppen haben nach Weinrich (ebd. 76ff.) die Funktion, „das Verhältnis von Textzeit und Aktzeit auszudrücken“. Als „Null-Stelle“ für die besprechende Tempus-Gruppe dient dabei das Präsens. Für die erzählende Tempus-Gruppe übernimmt diese Funktion das Präteritum ${ }^{2}$. Zusammenfassend lässt sich an dieser Stelle folgendes feststellen:

- Das deutsche Verbsystem verfügt über ein breites Spektrum an

2 Präsens und Präteritum dienen in beiden Tempus-Gruppen als „merkmallos“ (ebd.). (Weinrich 2001), die anderen Tempora weisen darauf hin, dass es „ein gewisses Maß an Aufmerksamkeit auf das Verhältnis von Textzeit und Aktzeit verwenden soll" und signalisieren damit den Rück- oder Vorausschau. 
Vergangenheitstempora. Das bereitet DaF-Lernern mit L1-Russisch besondere Schwierigkeiten, da es im Russischen nur ein Vergangenheitstempus gibt.

- Da die grammatische Kategorie des Aspekts im Russischen obligatorisch ist, ist das Tempussystem durch die Aspektopposition perfektiv/ imperfektiv gekennzeichnet. Dies führt dazu, dass Deutschlerner mit L1Russisch die Tendenz aufweisen, perfektive Situationen im Deutschen mit Perfekt und imperfektive Situationen mit Präteritum zu kodieren (Böttger 2008a).

- In Anlehnung an Slobin (1996), Stutterheim (2003, 2005) und Schmiedtová, Sahonenko (2008) wird davon ausgegangen, dass der grammatischen Kategorie des Aspekts hochautomatisierte Vorgänge zugrunde liegen, welche die Sprachproduktion bereits bei der Konzeptualisierung einer sprachlichen Äußerung beeinflussen.

- Die bisherigen empirischen Studien liefern starke Hinweise dafür, dass die Sprachproduktion russischsprachiger Deutschlerner sich im Bereich der Temporalität von den muttersprachlichen Daten deutlich unterscheidet. Sprecher mit L1-Russisch tendieren dazu, bei Nacherzählungen und Bildbeschreibungen andere Tempusentscheidungen im Deutschen zu treffen und weisen dabei einen Übergebrauch nicht-zielsprachlicher Präfigierung bei der Markierung der Abgeschlossenheit der Ereignisse im Deutschen auf.

\section{Fragestellungen und Hypothesen}

Die zentrale Fragestellung lautet:

- Welche Unterschiede gibt es bei der schriftlichen Beschreibung von Bildergeschichten durch deutsche Muttersprachler und fortgeschrittene russischsprachige Deutschlerner in der Verwendung von Verbformen und wie lassen sie sich erklären?

Anhand der Vorüberlegungen lassen sich folgende Subfragestellungen ableiten:

Fragestellung №1:

- Stimmt die Haupttempusform in der schriftlichen Textproduktion zur Bildbeschreibung bei russischsprachigen Deutschlernern und deutschen Muttersprachlern überein? 
Hypothese №1:

- In Bezug auf die bereits dargestellten Studien zur Perspektivierung der Ereignisse durch russischsprachige Deutschlerner ist zu erwarten, dass sie bei der schriftlichen Beschreibung eine Vergangenheitsperspektive wählen und sich für das Präteritum als Haupttempus entscheiden (Anstatt 2008, Schmiedtová, Sahonenko 2008).

Fragestellung №2:

- Gibt es Unterschiede in der Verwendung des Perfekts durch deutsche Muttersprachler und russischsprachige Deutschlerner?

Hypothese №2:

- Es ist denkbar, dass es bei der Experimentalgruppe mit russischsprachigen Deutschlernern zu einem Übergebrauch des Perfekts kommt (Smirnova 2009). Die muttersprachliche Strategie, perfektive Situationen durch perfektive Verbform zu markieren, welche durch das Vorhandensein der grammatischen Kategorie des Aspekts ermöglicht wird, kann sich auch in ihren Texten auf Deutsch niederschlagen.

Fragestellung №3:

- Weisen Texte von deutschen Muttersprachlern und russischsprachigen Deutschlernern auch Unterschiede in der Verwendung von telischen Verbpräfixen?

Hypothese №3:

- In Anlehnung an Schmiedtová, Sahonenko (2008) und Smirnova (2009) wird vermutet, dass bei der Markierung der Abgeschlossenheit der Situationen im Deutschen Sprachlerner mit L1-Russisch zu einem Übergebrauch von Verbpräfixen tendieren.

Fragestellung №4:

- Gibt es Unterschiede zwischen deutschen Muttersprachlern und russischsprachigen Deutschlernern in der Verwendung temporaler Adverbialen und temporaler Gliedsätze zum Ausdruck der Abgeschlossenheit im Deutschen?

Hypothese №4:

- Da Aspektualität im Deutschen lexikalisch realisiert wird, kann in Anlehnung an Böttger (2008a) und Schmiedtová, Sahonenko (2008) vermutet werden, dass Deutschlerner mit L1-Russisch die Strategie zur lexikalischen Markierung der temporalen Relationen nicht korrekt 
übernehmen. Ein Untergebrauch von temporalen Adverbien und temporalen Gliedsätzen zum Ausdruck der Abgeschlossenheit ist dabei zu erwarten.

\section{$3 \quad$ Methode}

\subsection{Design}

Der Schwerpunkt der Untersuchung liegt auf der Transkription einer visuell dargebotenen Situation anhand von Bildergeschichten des Tests Multilingual Assessment Instrument for Narratives (MAIN) (Gagarina, N., Klop D., Kunnari S., Tantele K., Välimaa K., Balciuniene I., Bohnhacker U., Walters J., 2012). Als Stimulusmaterial werden vier parallelisierte Geschichten dargestellt, die aus sechs Bildsequenzen bestehen. Wie in der Anleitung zu MAIN hingewiesen wird, sollte die Fragestellung so formuliert sein, dass die Testpersonen in ihrer Tempuswahl nicht manipuliert werden. Daher bekamen die Probanden die vier Bildergeschichten mit folgender Aufgabestellung: „Beschreiben Sie bitte die Bildergeschichte anhand von MAIN".

Die russischsprachigen Probanden erhielten nach dem Erledigen der Aufgabe auch die Fragebögen. Ziel war es, die Lernbiographie der russischsprachigen Probanden $\mathrm{zu}$ erforschen sowie einen Einblick in die muttersprachliche Textproduktion der Probanden zu ermöglichen. Die Fragen wurden dabei in drei Blöcken sortiert:

- Persönliche Fragen (Alter, Geschlecht etc.).

- Fragen zur Sprachbiographie (z.B. seit wann lernen die Probanden Deutsch etc.).

- Fragen zur Experimentaufgabe.

Im dritten Teil gab es eine Aufgabe zur Beschreibung der ersten drei Bilder von MAIN in der Muttersprache der Probanden. Wie aber in der Anweisung zur Testdurchführung anhand von MAIN hervorgehoben wurde, sollte bei bilingualen bzw. mehrsprachigen Probanden der Zeitraum zwischen den Testungen 4 bis 7 Tage betragen, um sprachübergreifende Einflüsse zu verringern. Aus diesem Grund wurden den Probanden der Experimentalgruppe die Fragebögen nicht gleich, sondern erst nach 4 Tagen zum Ausfüllen zugeschickt. Diese Fragebögen wurden nur an die Probanden mit L1-Russisch verteilt. Die Studenten mit L1Deutsch hatten die Beschreibung der MAIN-Bilder als Hausaufgabe bekommen. Erst danach wurden ihnen in einem Seminar die Einverständniserklärungen verteilt, mit deren Signierung sie der Nutzung ihrer Daten für die vorliegende 
Studie zustimmten. Die möglichen regionalen Unterschiede innerhalb Deutschlands, Österreichs oder der deutschsprachigen Schweiz wurden für die Ziele der Untersuchung nicht als Störfaktor gesehen.

\subsection{Probanden}

In der Studie wurden die schriftlichen Daten von insgesamt 16 Studienteilnehmern analysiert. Neben den Texten von acht fortgeschrittenen Deutschlernern mit L1-Russisch wurden auch für die Analyse die Vergleichsdaten von acht Muttersprachlern herangezogen.

Das Durchschnittsalter der muttersprachlichen Probanden lag bei 26,1 Jahren. Der jüngste Proband war 20 Jahre, der älteste 49 Jahre alt ${ }^{3}$. Die Mehrheit der Probanden (sieben Personen) war weiblich, der männliche Anteil lag bei einem Probanden. Alle studieren Germanistische Linguistik an der HumboldtUniversität zu Berlin. Für die Probandengruppe mit L1-Russisch wurden fortgeschrittene Deutschlerner mit L1-Russisch ausgesucht, die Deutsch im gesteuerten ${ }^{4}$ DaF-Unterricht erworben haben.

Das Durchschnittsalter der Probanden mit L1-Russisch lag bei 27,7 Jahren. Alle Probanden mit L1-Russisch haben einen akademischen Hintergrund. Bei den meisten Probanden war Deutsch die erste Fremdsprache. Alle Probanden hatten entweder die Niveaustufe C1 oder C2. Bei allen Probanden begann der Erwerb des Deutschen vor der Pubertät. Das war entscheidend für die Auswahl der Probanden, da laut der Critical Period Hypothesis „die Erwerbsunterschiede zwischen frühen und späten Lernern auf die Altersgebundenheit neurobiologisch determinierter ,Zeitfenster"“ (Pagonis, 2009: 47) zurückzuführen sind.

\subsection{Durchführung}

Die Pilotierungsphase erfolgte in zwei Schritten. Zunächst wurden die Vortests mit den russischsprachigen Deutschlernenden durchgeführt, die nach eigener Einschätzung Deutschkenntnisse auf den Niveaustufen B1-B2 (GeR) hatten. Die Tests wurden per E-Mail durchgeführt.Für viele Versuchspersonen erwies sich jedoch diese Aufgabe als sehr zeitaufwendig und anspruchsvoll. Das könnte eventuell dadurch erklärt werden, dass die Probanden noch unsicher in der Wiedergabe der deutschen Tempora sowie der temporalen Gliedsätze waren.

3 Obwohl die Altersgrenze für die Teilnehmer zwischen 20 und 35 Jahren alt lag, wurde für diesen Probanden eine Ausnahme gemacht. Der Grund dafür war die Tatsache, dass es in diesem Fall um die muttersprachlichen und nicht L2-Daten ging.

4 In Anlehnung an Stutterheim (1986: 26) wird damit der Begriff „gesteuert“ für „den Spracherwerb mit unterrichtlicher Unterweisung“ verstanden. 
Im weiteren Schritt wurde entschieden, eine andere Experimentalgruppe mit Deutschkenntnissen auf C1-Niveau (GeR) zu testen. Es wurden die schriftlichen Daten von acht weiteren Testpersonen, die L1-Russisch haben und einen C1-Kurs in Sprachenzentren der Humboldt-Universität zu Berlin (HU) und der Freien Universität Berlin (FU) belegten, erhoben. Die Daten wurden nicht per E-Mail, sondern während persönlicher Treffen in den Räumen der jeweiligen Universität erhoben. Doch auch die zweite Experimentalgruppe schien von der Aufgabe zur Bildbeschreibung überfordert zu sein. Viele Teilnehmer waren mit lexikalischen Schwierigkeiten konfrontiert und bildeten mehrheitlich einfache Sätze. Letztendlich wurde entschieden, in der tatsächlichen Untersuchung die Teilnehmergruppe der Niveaustufe C1-C2 zu testen und die Daten schriftlich per E-Mail zu erheben.

Im nächsten Schritt erfolgte die Datenerhebung der russischsprachigen Probanden. Die Probanden erhielten eine E-Mail auf Russisch mit einer kurzen Danksagung für die Teilnahme und der Bitte die Einverständniserklärung zu unterschreiben. Des Weiteren wurden sie gebeten, die auf Deutsch formulierte Aufgabestellung zu erledigen. Um die möglichen sprachlichen Unterschiede bei der Übersetzung ins Russische zu vermeiden und damit die Gleichheit der Bedingungen für die beiden Experimentalgruppen sicherzustellen, wurde die Aufgabestellung für die russischen Probanden ebenso auf Deutsch formuliert. Die Probanden wurden gebeten, die Aufgabe möglichst spontan und ohne Unterbrechung schriftlich auszuführen und zurückzuschicken.

\section{$4 \quad$ Ergebnisse}

Die Ergebnisse der Studie wurden in fünf Schritten analysiert.

\subsection{Datenauswertung zum Tempusgebrauch}

Im ersten Schritt wurden die erhobenen Texte in Bezug auf die Wahl der Haupttempusform analysiert. Dafür wurde die Gesamtzahl der finiten Verbformen in der Textproduktion der Kontrollgruppe und der Experimentalgruppe ermittelt und die temporale Verteilung analysiert. Die Datenanalyse der muttersprachlichen Daten ergab ein einheitliches Bild: alle deutschen Muttersprachler entschieden sich bei der Beschreibung der MAINBilder für das Präsens als Haupttempusform. Die überwiegende Mehrheit in der russischsprachigen Experimentalgruppe wählte hingegen eine andere temporale Perspektive auf die Bildergeschichten und benutzte Präteritum als Haupttempusform. In Tabelle 1 sind die Ergebnisse anschaulich zusammengebracht: 
Tabelle 1: Verteilung der Hauptempusformen in den Texten der Kontrollgruppe und der Experimentalgruppe

\begin{tabular}{|l|c|c|c|c|c|c|c|c|}
\hline L1-Deutsch & DP1 & DP2 & DP3 & DP4 & DP5 & DP6 & DP7 & DP8 \\
\hline Haupttempus & Präs & Präs & Präs & Präs & Präs & Präs & Präs & Präs \\
\hline L1-Russisch & RP1 & RP2 & RP3 & RP4 & RP5 & RP6 & RP7 & RP8 \\
\hline Haupttempus & Prät & Prät & Prät & Prät & Präs & Prät & Prät & Präs \\
\hline
\end{tabular}

Aus der Tabelle 1 ist ersichtlich, dass sich zwei Probandinnen mit L1-Russisch für Präsens als Haupttempusform entschieden ${ }^{5}$, die Anderen benutzten Präteritum. Insgesamt verwendeten die deutschen Muttersprachler 571 finite Verbformen. Die temporale Verteilung sah dabei wie folgt aus:

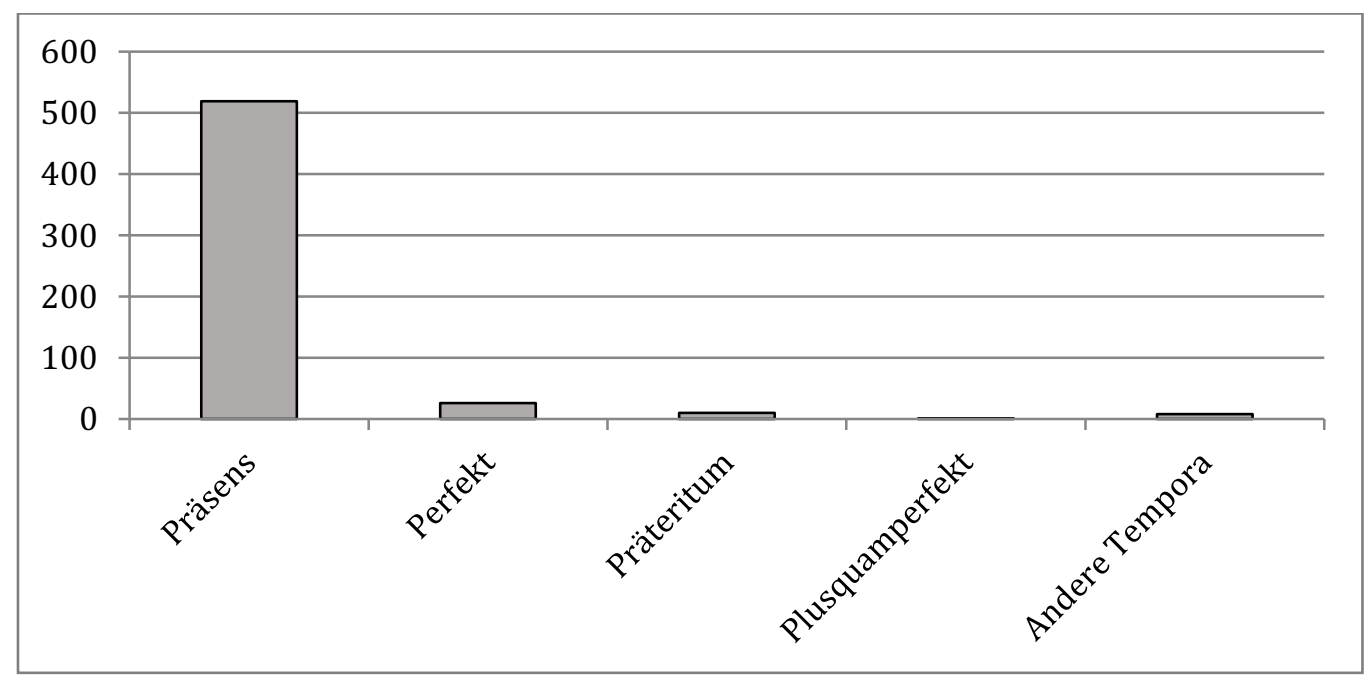

Abbildung 1: Verteilung der Tempora bei L1-Deutsch

Folgender Satz war für die Textproduktion der deutschen Muttersprachler typisch:

(1) Eine Katze jagt vergebens einen Schmetterling und resigniert, nachdem sie sich in den Dornen eines Busches verfangen hat. (DP2)

Aus (1) ist ersichtlich, dass im Hauptsatz die Topikzeit mit der Äußerungszeit zusammenfällt. Zum Ausdruck der Vorzeitigkeit wird dabei im Nebensatz das Perfekt gebraucht.

Ein anderes Bild ergibt sich bei der Analyse der deutschen Texte von russischsprachigen Probanden. Insgesamt verwendeten Studienteilnehmer mit

5 Dies ist auch ein Thema im nächsten Abschnitt, da die Analyse der Fragebogen berücksichtigt werden muss. 
L1-Russisch 498 finiten Verbformen. Die temporale Verteilung sah in der Experimentalgruppe folgendermaßen aus:

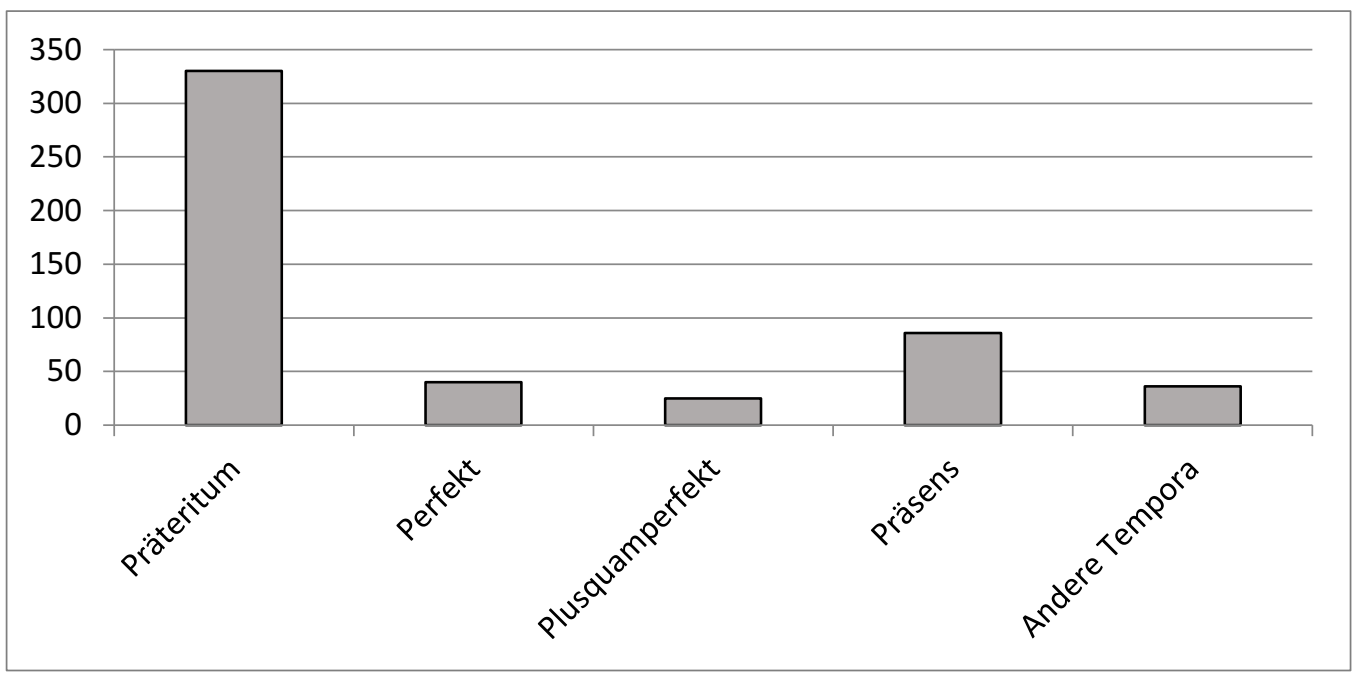

Abbildung 2: Verteilung der Tempora bei L1-Russisch

Folgender Satz macht die Präferenz der russischsprachigen Deutschlerner anschaulich:

(2) Die glückliche Mutter, die gerade ihr Kind gerettet hatte, lenkte sich ab, in diesem Moment sprang der Fuchs hinter dem Baum hervor. (RP1)

In (2) verlegt die Probandin im Hauptsatz die Topikzeit vor der Äußerungszeit, das Plusquamperfekt markiert dabei die Vorzeitigkeit eines Ereignisses im Nebensatz.

Zusammenfassend kann man festhalten, dass die Kontrollgruppe mit L1Deutsch und die Experimentalgruppe mit L1-Russisch sich für verschiedene temporale Relationen entschieden haben: deutsche Muttersprachler wählten Präsens als Haupttempus für ihre Bildergeschichten. Die Sprechzeit fiel in ihren Texten mit der Topikzeit zusammen (TU in TT). Russische Muttersprachler verlegten hingegen die Topikzeit vor die Äußerungszeit, (TT vor TU) und bildeten dabei Sätze mit dem Vergangenheitsbezug. Als Haupttempus galt dabei das Präteritum.

\subsection{Datenauswertung der Fragebögen der russischsprachigen Experimentalgruppe}

Im nächsten Schritt wurden die Fragebögen der russischsprachigen Probanden evaluiert. Der Fokus der Analyse lag auf dem dritten Block der Fragen, welcher sich unmittelbar auf das Experiment bezog. Die Probanden wurden gebeten, die 
Wahl der Haupttempusform in ihren auf Deutsch verfassten Texten zu begründen und danach die ersten drei Bilder von den MAIN-Bildergeschichten in ihrer Muttersprache $\mathrm{zu}$ beschreiben. Es stellte sich heraus, dass die Mehrheit der Probanden (5 von 8) in ihren muttersprachlichen Texten perfektive Vergangenheitsform wählte. Diese Erkenntnis stimmt auch mit den Ergebnissen der Studie von Anstatt (2008) überein, wonach Probanden mit L1-Russisch in ihren muttersprachlichen Texten die perfektive Vergangenheitsform als Haupttempusform benutzten. In diesem Zusammenhang ist auch interessant, dass zwei von den drei Probandinnen mit L1-Russisch, welche in ihren Texten auf Deutsch das zielsprachliche Präsens wählten, auch in ihren russischen Texten dieselbe Strategie verfolgten und sich für die imperfektive Verbform im Präsens entschieden (RP5, RP8). Bei der dritten Probandin (RP3) unterschied sich die Haupttempusform in den beiden Texten: im deutschen Text benutzte sie das Präteritum und im Russischen das Präsens. Im nächsten Schritt wurden die Begründungen der Wahl der Haupttempusform analysiert. Aus Tabelle 2 ist ersichtlich, dass für die meisten Probanden das Stichwort „Erzählung“ ausschlaggebend war, um sich für das Präteritum zu entscheiden.

Tabelle 2: Begründung der Wahl der Haupttempusform durch Probanden mit L1-Russisch

\begin{tabular}{|l|l|}
\hline RP1 & „Weil sie am besten für eine Erzählung passt“. \\
\hline RP2 & „Es geht um eine zusammenhängende Erzählung in der Vergangenheit“. \\
\hline RP3 & „Es geht um eine Geschichte in der Vergangenheit, die ich erzähle“. \\
\hline RP4 & $\begin{array}{l}\text { "Ich habe die Geschichte im Präteritum beschrieben, weil das eine Nacherzählung war } \\
\text { Geschichte erzählt“. }\end{array}$ \\
\hline RP5 & „Ich war davon ausgegangen, es handelt sich um jetzt“. \\
\hline RP6 & „Das ist die Hauptzeitform der erzählten Rede“. \\
\hline RP7 & $\begin{array}{l}\text { „Ich habe die Bilder in Form von einer zusammenhängenden Erzählung beschrieben }- \\
\text { wie bei einem Märchen. So passt Präteritum am besten“. }\end{array}$ \\
\hline RP8 & $\begin{array}{l}\text { „Die Bilder sehen wie Kinderbilder aus und für die Kinder würde ich alles in Präsens } \\
\text { beschreiben, denn es ist erstens leichter für sie zu verstehen, zweitens dient es zur } \\
\text { Dynamik und macht die Erzählung spannender“. }\end{array}$ \\
\hline
\end{tabular}

An dieser Stelle sei angemerkt: Einige der fortgeschrittenen Deutschlerner mit L1-Russisch, die im zweiten Pilotierungsgang getestet wurden und mehrheitlich die Texte im Präsens auffassten (RP5 und RP8), gaben in ihren Fragebogen folgende Antwort auf diese Frage: „Weil es um Hier und Jetzt geht“. Dies wird später in der Diskussion aufgegriffen. 


\subsection{Datenauswertung zur Verwendung des Perfekts}

Nach Smirnova (2009) tendieren russischsprachige Deutschlerner dazu, perfektive Situationen mit dem Perfekt zu kodieren. Als Beispiel dafür dienen die Daten einer Probandin mit L1-Russisch, welche in der Pilotierungsphase erhoben wurden. Für ihre Bildbeschreibung wählte die Probandin das Perfekt als Haupttempusform mit wenigen Übergängen zum Präteritum, wenn es um imperfektive Situationen ging. Da die Probandin Deutsch erst seit drei Jahren lernt, kann man mit hoher Wahrscheinlichkeit annehmen, dass sie ins Deutsche die muttersprachliche Präferenz, perfektive Situationen mit Perfekt und imperfektive mit Präteritum zu kodieren, direkt ins Deutsche übertragen hat.

Dementsprechend wurde entschieden, im dritten Analyseschritt die Verwendung des Perfekts durch die Kontrollgruppe und die Experimentalgruppe zu analysieren. Wie aber im Analyseschritt №1 dargestellt wurde, wählten beiden Gruppen unterschiedliche temporale Perspektiven auf die Bildergeschichten: Während deutsche Muttersprachler sich für das Präsens als Haupttempus entschieden, bildeten Deutschlerner mit L1-Russisch die Sätze mit Vergangenheitsbezug, im Präteritum. Nach Weinrichs Tempusmodell dient das Perfekt als Relation zum Präsens beim Ausdruck der Vorzeitigkeit. Dieselbe Funktion übernimmt auch das Plusquamperfekt für das Präteritum. Die unterschiedlichen temporalen Relationen, welche die Probanden mit L1-Russisch und L1-Deutsch in ihren Texten wählten, machten die Vergleichsanalyse der Kontrollgruppe und der Experimentalgruppe in diesem Schritt unmöglich.

Auf Grund dessen wurde in diesem Analyseschritt folgenderweise vorgegangen: Zunächst wurden alle Perfekt- und Plusquamperfektmarkierungen durch beide Gruppen zusammengefasst und gezählt. Danach wurde der relative Gebrauch der Tempora zum Ausdruck der Vorzeitigkeit aussortiert ${ }^{6}$.

Da der Fokus der Analyse auf dem konzeptuellen Gebrauch des Perfekts durch russischsprachige Deutschlerner lag, wurden im nächsten Schritt nur jene Fälle analysiert, in denen das Perfekt durch die Experimentalgruppe mit L1Russisch als „Konkurrenz“ zum Präteritum gebraucht wurde. Die Daten wurden ebenso mit einer deutschen Muttersprachlerin, die DaF an der HU studiert, in Bezug auf den native-like Gebrauch durch Probanden mit L1-Russisch diskutiert. Die Datenanalyse zum Perfektgebrauch der Experimentalgruppe hat gezeigt, dass

6 An dieser Stelle sei angemerkt, dass die Datenanalyse zum relativen Gebrauch keine Unterschiede gezeigt hat. Die deutschen Muttersprachler verwendeten Perfekt als Relation zum Präsens, um die Vorzeitigkeit der Ereignisse zu markieren. Genau das machten auch die Deutschlerner mit L1-Russisch, um mit dem Plusquamperfekt die vorzeitige Relation zum Präteritum herzustellen. Interessant ist allerdings, dass alle Plusquamperfektformen (insgesamt 25 Treffer) in den Texten von nur zwei besonders fortgeschrittenen Probandinnen zu finden sind. 
in fast der Hälfte der Fälle im Deutschen die nicht-zielsprachliche Präferenz gewählt wurde.

Insgesamt verwendeten die Probanden, welche ihre Texte im Präteritum als Haupttempusform verfassten, 32 Perfektmarkierungen. In 14 Fällen wurde dabei das Perfekt entweder anstelle des Plusquamperfekts zur Herstellung der Vorzeitigkeit benutzt oder alternativ zum Präteritum, was von einer deutschen Muttersprachlerin als „ist möglich, Präteritum wäre aber besser“ eingestuft wurde. In Abbildung 3 sind die Ergebnisse anschaulich dargestellt:

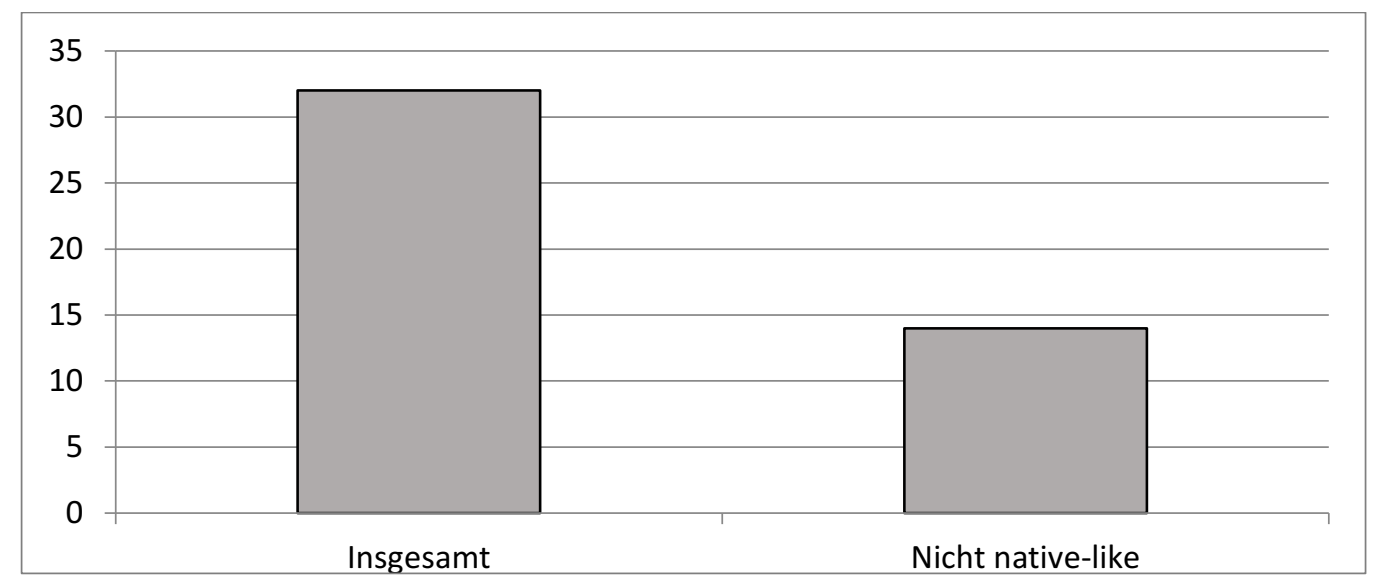

Abbildung 3: Perfektmarkierungen durch die Experimentalgruppe mit L1-Russisch

Auffallend ist, dass in 12 von 14 Fällen die russischsprachigen Probanden damit den perfektiven Charakter Nicht native-like zu realisieren versuchten. Folgendes Beispiel (3) ist dabei für diese Präferenz der Deutschlerner mit L1-Russisch besonders anschaulich:

(3) Inzwischen ist der Kater neben dem Nest erschienen und wollte die kleinen jungen Vögelchen aufessen, aber da ist ein Hund herbeigelaufen und den Kater weggetrieben. (RP6)

Zum Ausdruck der Imperfektivität wird dabei in (3) das Präteritum gewählt, während die perfektiven Situationen mit Perfekt realisiert sind. Es sei aber zu betonen, dass dies von der deutschen Muttersprachlerin nicht als grammatischer Fehler gesehen wurde, sondern als zweitbeste Wahl.

\subsection{Datenauswertung zur Verwendung von telischen Präfixen}

In Anlehnung an Schmiedtová/ Sahonenko (2008) wird in der vorliegenden Studie davon ausgegangen, dass bei der Markierung der Abgeschlossenheit der Situationen im Deutschen Deutschlerner mit L1-Russisch zu einem Übergebrauch von Verbpräfixen tendieren. Außerdem wird in Anlehnung an Smirnova (2009: 


\section{russischsprachiger Deutschlerner}

49) vermutet, dass beim Ausdruck der „Raumverhältnis-Konzepte“ (ebd.) Deutschlerner mit L1-Russisch Verben mit telischen Präfixen „als ein Perfektivierungsmittel zum Ausdruck der Abgeschlossenheit“" interpretieren.

Dementsprechend wurden im vierten Schritt der Datenanalyse alle finiten Verben mit telischen Präfixen beider Gruppen zusammengefasst und analysiert. Diese Aufgabe wurde allerdings dadurch erschwert, dass viele Präfixe auch als „Wortbildungsmittel“ (Helbig/ Buscha, 2001: 63ff.) der perfektiven ${ }^{7}$ Aktionsarten dienen. Die Analyse der inhärenten temporalen Eigenschaften gehörte jedoch nicht zum Gegenstand dieser Arbeit. Dementsprechend wurden nur die telischen Präfixe mit räumlicher Bedeutung mitgezählt und danach prozentual im Hinblick auf die Gesamtzahl aller finiten Verbformen berechnet. $\mathrm{Zu}$ telischen Präfixen mit räumlicher Bedeutung gehörten dabei: ein-, aus-, auf-, unter- ${ }^{8}$, durch-, los-, hoch-, weg-, davon-, ver-, ab-, fort-. In Abbildung 4 sind die Ergebnisse der Datenauswertung anschaulich zusammengebracht.

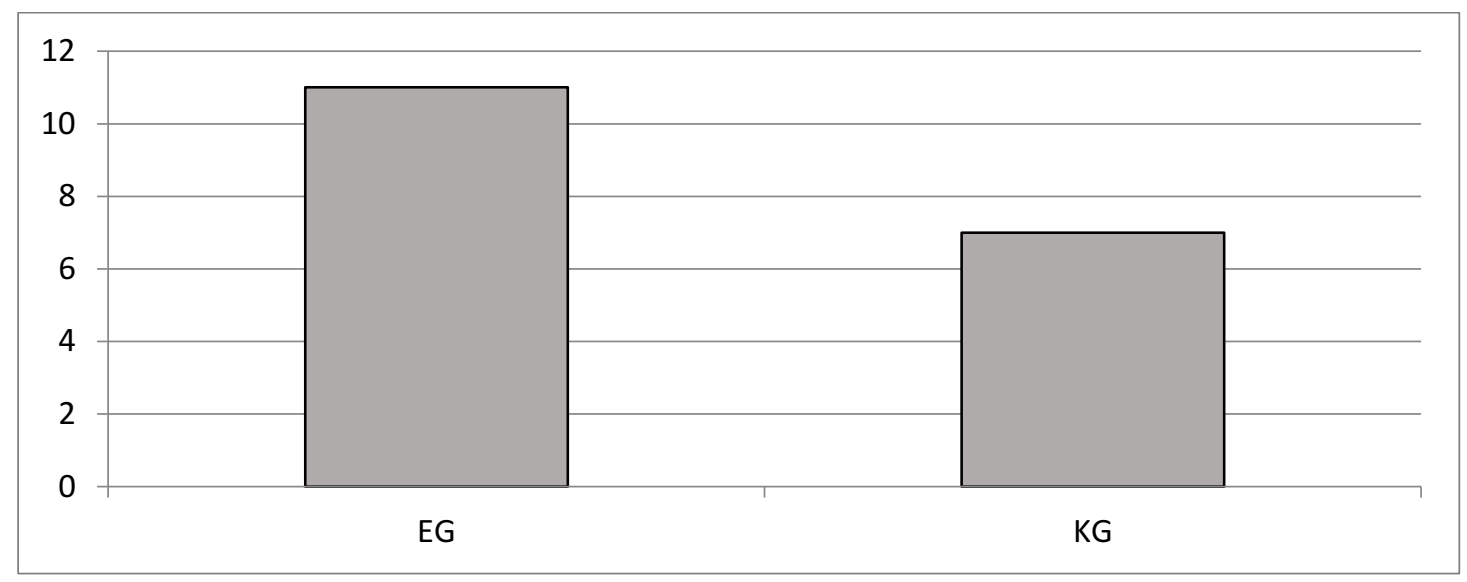

Abbildung 4: Gebrauch von telischen Präfixen durch die Kontrollgruppe (KG) und die Experimentalgruppe (EG)

Wie aus Abbildung 4 ersichtlich ist, verwendeten die deutschen Muttersprachler mehr Präfixe mit telischer Bedeutung als Probanden mit L1-Russisch. Der Unterschied ist jedoch nicht sehr groß.

\subsection{Datenauswertung zur Verwendung von temporalen Adverbialen}

Da der Aspekt im Deutschen lexikalisch realisiert wird, ist es für die Ziele der vorliegenden Studie wichtig, die lexikalischen Mittel zum Ausdruck der Temporalität zu analysieren. In diesem Abschnitt soll der Gebrauch von

7 Nach der Verbklassifikation von Helbig/Buscha unterscheidet man zwischen den durativen und perfektiven Aktionsarten, die ihrerseits auch eigene Unterklassen aufweisen. Vgl. dazu: Helbig/Buscha, 2001: 62ff.

8 Auch heraus-, hinauf- und herunter- 
temporalen Adverbialien zum Ausdruck der Abgeschlossenheit im Deutschen durch die Kontrollgruppe mit L1-Deutsch und die Experimentalgruppe mit L1Russisch verglichen werden. Die im Kapitel 3 aufgestellte Hypothes besagt, dass die Probanden mit L1-Russisch die zielsprachliche Tendenz nicht erkennen und die Abgeschlossenheit von Situationen durch lexikalische Mittel markieren. Der Fokus der Analyse wurde dabei auf temporale Adverbiale und temporale Gliedsätze gelegt, die zum Ausdruck der Abgeschlossenheit dienen.

In Anlehnung an Böttger (2008a) wurden folgende temporale Adverbiale zum Ausdruck der Perfektivität gezählt:

- Adverbien oder adverbiale Bestimmungen, welche die Begrenzung einer Handlung anzeigen: plötzlich, gleich, ganz, vollends, innerhalb einer Stunde etc.

- Folgende adverbiale Gliedsätze: ehe, bevor, nachdem, bis.

Die Datenanalyse hat gezeigt, dass sowohl die Kontrollgruppe als auch die Experimentalgruppe generell sehr wenig Adverbien und adverbiale Gliedsätze zum Ausdruck der Abgeschlossenheit gebrauchten. Deutsche Muttersprachler verwendeten in ihren Texten insgesamt drei Adverbien bzw. adverbiale Gliedsätze, russischsprachige Deutschlerner gebrauchten elf. Der Unterschied ist nicht so groß.

\section{Diskussion}

Die durchgeführte empirische Studie hatte sich zum Ziel gesetzt, die schriftliche Textproduktion der deutschen Muttersprachler und der Deutschlerner mit L1Russisch im Bereich von Temporalität und Aspektualität im Hinblick auf den möglichen Transfer aus der L1 zu analysieren.

Die Analyse hat gezeigt, dass deutsche Muttersprachler und Deutschlerner mit L1-Russisch unterschiedliche temporale Perspektiven auf die Bildergeschichten einnahmen. Deutsche Muttersprachler wählten bei der Beschreibung der Bildergeschichten die temporale Referenz der Gegenwart (Sprechzeit in der Topikzeit) und gebrauchten dafür das Präsens. Die vergangenen Sachverhalte wurden dabei mit Perfekt markiert. Russischsprachige Deutschlerner wählten die Zeitreferenz der Vergangenheit (Topikzeit vor der Sprechzeit) und entschieden sich dabei für das Präteritum als Haupttempusform. Die Auswertung der Fragebögen der Experimentalgruppe mit L1-Russisch hat gezeigt, dass die Wahl des Präteritums als Haupttempusform von den Probanden durchaus reflektiert wurde. Für sie ging es in der Aufgabe zur Bildbeschreibung um eine Erzählung. Da diese Erzählung außerdem schriftlich verfasst werden 
sollte, war für sie anscheinend die Wahl des Präteritums selbstverständlich, da das Präteritum traditionell als Erzähltempus der schriftlichen Rede gilt.

Dennoch lassen die Stichproben der muttersprachlichen Texte der Probanden mit L1-Russisch, sowie die Evidenzen aus den vorherigen Untersuchungen (Anstatt 2008, Schmiedtová/ Sahonenko 2008) vermuten, dass es zum Transfer der muttersprachlichen Präferenz im Bereich des Tempus kommen könnte, und zwar aus folgendem Grund: Auch in ihren Texten auf Russisch wählte die Mehrheit der Probanden die Vergangenheitsperspektive TT vor TU. Diese Strategie wird im Russischen auf doppelte Weise begünstigt. Zum einen schafft das Vorhandensein der grammatischen Kategorie des Aspekts im Russischen die Aspektopposition perfektiv/imperfektiv. Wie Anstatt (2008: 21) hinweist: „Especially in narrations, aspect has a quite central function: The perfective aspect marks a sequence of two situations and thus is said to drive forward the action".

Zum anderen weist das russische Präsens eine Lücke auf: Der Gegenwartsbezug mit der perfektiven Form trägt meistens den Zukunftscharakter und ist dadurch mit telischen Verben inkompatibel (vgl. Krifka 1989). Man könnte vermuten, dass diese Besonderheiten des russischen Tempus- und Aspektsystems dazu führen, dass die temporale Perspektive der Topikzeit vor der Äußerungszeit im Russischen als besonders bevorzugt gilt.

Die Probanden der zweiten Testgruppe verfassten ihre Texte hingegen im Präsens. Die Datenerhebung erfolgte während persönlicher Treffen und die Autorin war während der Aufgabenerfüllung anwesend. Häufig begründeten die Probanden ihre Entscheidung für das Präsens als Haupttempus wie folgt: , Es geht um Hier und Jetzt". Man könnte annehmen, dass für diese Probandengruppe eine solche Erhebungssituation womöglich eine „gespannte Sprechhaltung“ (Weinrich, 2001) suggerierte, die sie dazu bewogen hat, ihre Texte im Präsens zu verfassen, während die Datenerhebung per E-Mail als „entspannt“ wahrgenommen wurde und demzufolge zu einer erzählenden Perspektive einlud. Dies würde mit Weinrichs Einteilung der deutschen Tempora in zwei Gruppen übereinstimmen. Wie Böttger (2008a: 126) betont, gehört die Überproduktion des historischen Präsens zu einem der häufigsten Fehler bei russischsprachigen Deutschlernern. Die mögliche Ursache dafür sieht Böttger ,in der intralingual bedingten Unsicherheit bei der Bildung der deutschen Vergangenheitsformen“.

Die Datenanalyse zum Perfektgebrauch durch die Experimentalgruppe mit L1-Russisch hat gezeigt, dass die Probanden mit L1-Russisch fast in der Hälfte der Fälle in ihrer Wahl entweder eine grammatisch falsche oder eine nichtmuttersprachliche Entscheidung getroffen haben. Entweder gebrauchten sie Perfekt statt Plusquamperfekt zum Ausdruck der Vorzeitigkeit oder versuchten durch Perfekt im Deutschen den perfektiven Charakter der Situationen 
auszudrücken, was in den meisten Fällen auch mit Präteritum möglich (und in vielen Fällen sogar besser) gewesen wäre.

Die Verteilung der telischen Verbpräfixe zwischen der Kontrollgruppe und der Experimentalgruppe war zwar bei deutschen Muttersprachlern sogar ein bisschen höher (11\% gegenüber 7\% bei Probanden mit L1-Russisch). Es sei an dieser Stelle jedoch noch einmal hervorzuheben, dass nur die telischen Verbpräfixe mit räumlicher Bedeutung mitberechnet wurden, da die Analyse der Aktionsarten nicht zum Ziel der vorliegenden Studie gehörte.

Obwohl lexikalische Mittel zum Ausdruck der Temporalität in der Lernersprache vor den morphologischen Mitteln erscheinen, zeigte die Analyse der erhobenen Daten allerdings keinen signifikanten Unterschied zwischen den beiden Gruppen im Gebrauch temporaler Adverbialen und temporaler Gliedsätze zum Ausdruck der Abgeschlossenheit. Die Probanden aus beiden Gruppen verwendeten generell sehr wenige solcher Mittel. Die Mittel zum Ausdruck der Imperfektivität waren nicht Gegenstand der vorliegenden Studie.

\section{$6 \quad$ Fazit und Ausblick für den DaF-Unterricht}

Die vorliegende Studie verfolgte das Ziel, den Einfluss von russischem Tempusund Aspektsystem auf die Wahl der Verbformen im Deutschen anhand schriftlicher Textproduktionen der Deutschlerner mit L1-Russisch zu analysieren. Im Rahmen einer Fallstudie wurde die schriftliche Textproduktion deutscher Muttersprachler und Deutschlerner mit L1-Russisch verglichen und analysiert. Die zentrale Fragestellung dabei lautete:

- Gibt es bei der schriftlichen Beschreibung von Bildergeschichten durch deutsche Muttersprachler und fortgeschrittene russischsprachige Deutschlerner Unterschiede in der Verwendung von Verbformen?

Es ließ sich feststellen, dass es der Experimentalgruppe mit L1-Russisch nicht gelungen ist, Texte auf Deutsch $\mathrm{zu}$ fassen, die ,den muttersprachlichen Intuitionen" (Stutterheim/ Carroll, 2005: 1) entsprechen. Zum einen wählten die russischsprachigen Deutschlerner in ihrer Textproduktion eine „erzählende“ Perspektive auf die Bildergeschichten: Sie verlegten den Fokus des Textes in die Vergangenheit und benutzten das Erzähltempus Präteritum. Die deutschen Muttersprachler bildeten dagegen Sätze mit Gegenwartbezug, indem sie das Präsens als Haupttempusform benutzten und damit eine „besprechende“ Perspektive einnahmen.

Der Unterschied in den temporalen Perspektiven zwischen der Testgruppe mit L1-Russisch (Präsens als Haupttempusform) und der Experimentalgruppe mit L1-Russisch (Präteritum als Haupttempusform) aufgrund der unterschiedlichen 
Erhebungsbedingungen lassen sich mithilfe von Weinrichs Gruppierung der deutschen Tempora gut erklären. Dies könnte in Anlehnung an Schumacher (2005) zur Unterscheidung zwischen Präteritum und Perfekt (sowie auch Plusquamperfekt) auch für russische Deutschlerner herangezogen werden.

Bei der Analyse ausgewählter sprachlicher Mittel zum Ausdruck der Abgeschlossenheit im Deutschen (Perfektmarkierungen, temporale Adverbiale und temporale Gliedsätze) stellte sich heraus, dass Deutschlerner mit L1-Russisch die zielsprachlichen Präferenzen im Deutschen nicht immer erkennen. Perfekt wurde in den meisten Fällen zwar grammatisch richtig gebraucht, war jedoch die sekundäre Wahl nach dem Präteritum. Die Probanden mit L1-Russisch versuchten den abgeschlossenen Charakter der Situationen auszudrücken, indem sie die perfektiven Lesarten des Präteritums außer Acht ließen.

Der Vergleich des Gebrauchs telischer Präfixe ergab keinen signifikanten Unterschied. Bei der Verwendung temporaler Adverbialen und temporaler Gliedsätze zum Ausdruck der Abgeschlossenheit konnte ebenso kein Unterschied festgestellt werden.

Diese Unterschiede lassen sich durch Transfererscheinungen aus der Muttersprache der Probanden mit L1-Russisch erklären. Vor allem der obligatorische grammatische Aspekt im Russischen sorgte dafür, dass die Probanden mit L1-Russisch im Deutschen primär nach den grammatischen Mitteln zum Ausdruck der Abgeschlossenheit gesucht haben (Perfektmarkierungen).

Im Hinblick auf die weitere Forschung wäre es interessant, die Ergebnisse dieser empirischen Untersuchung anhand mündlicher Daten zu prüfen. Das Präteritum gilt als Erzähltempus, vor allem aber in der Schriftsprache. Es wäre deswegen interessant zu schauen, für welche temporale Perspektive sich die Probanden mit L1-Russisch bei der mündlichen Bildbeschreibung entscheiden würden. Die mündliche Sprachproduktion ist auch durch ihren spontanen Charakter gekennzeichnet und es wäre spannend zu untersuchen, ob die Lernersprache der Probanden mit L1-Russisch mehr Transfererscheinungen aus ihrer L1 aufweist.

Es wäre außerdem aufschlussreich, die inhärenten lexikalischen Eigenschaften der benutzten Verben bzw. der Situationstypen zu analysieren. Da „der Ausdruck eines Situationstypen ein Phänomen an der Schnittstelle von Lexikon und Grammatik“ (Schumacher, 2005: 151) ist, wäre es interessant zu prüfen, ob russischsprachige Deutschlerner zu einem Übergebrauch von telischen Situationstypen (Achievements und Accomplishments nach der Vendlerschen Klassifikation) im Deutschen tendieren. Man könnte erwarten, dass sie auf solche Weise im Deutschen den Ausdruck der grammatischen Perfektivität aus ihrer Muttersprache realisieren. 
Zusammenfassend lässt sich festhalten, dass das russische Tempus- und Aspektsystem einen erkennbaren Einfluss auf die Wahl der Verbformen im Deutschen durch russischsprachige Deutschlerner ausübt. Dies sollte in einem DaF-Unterricht für Deutschlerner mit L1-Russisch sowie in der DaF-Didaktik berücksichtigt werden.

\section{$7 \quad$ Literaturverzeichnis}

Anstatt, T. (2008). Aspect and tense in storytelling by Russian, German and bilingual children. Russian Linguistics 32(1): 1-26.

Böttger, K. (2008a). Die häufigsten Fehler russischsprachiger Deutschlerner. Ein Handbuch für Lehrende. Münster, New York, München, Berlin: Waxmann.

Böttger, K. (2008b). Negativer Transfer bei russischsprachigen Deutschlernern Die häufigsten muttersprachlich bedingten Fehler vor dem Hintergrund eines strukturellen Vergleichs des Russischen mit dem Deutschen. Hamburg: Universität Hamburg.

Bardovi-Harlig, K. (2000). Tense and aspect in second language acquisition: form, meaning, and use. Malden, MA: Blackwell.

Bardovi-Harlig, K. (2007). One Functional Approach to Second Language Acquisition: The Concept-Oriented Approach. In: B. Van Patten / J. Williams Theories in Second Language Acquisition. An Introduction, 57-77. New Jersey: Lawrence Erlbaum Associates, Inc., Publishers.

Carroll, M. \& Lambert M. (2003). Information structure in narratives and the role of grammaticised knowledge: A study of adult French and German learners of English. In: C. Dimroth / M. Starren Information Structure and the Dynamics of Language Acquisition, 267-287. Amsterdam: John Benjamins.

Comrie, B. (1985). Tense. Cambridge: Cambridge University Press.

Gagarina, N., Klop D., Kunnari S., Tantele K., Välimaa K., Balciuniene I., Bohnhacker U. \& Walters J. (2012) Multilingual Assessment Instrument for Narratives. ZAS Papers in Linguistics, 56.

Klein, W. (1994). Time in Language. London: Routledge.

Klein, W. (1995). Frame of analysis. In: R. Dietrich/ W. Klein/ C. Noyau The acquisition of temporality in a second language, 17-29. Amsterdam/Philadelphia: John Benjamins.

Klein, W. (2009). The expresison of time. Berlin: Mouton de Gruyter.

Krifka, M. (1989) Nominalreferenz und Zeitkonstitutionen. Zur Semantik von Massentermen, Pluraltermen und Aspektklassen. München: Wilhelm Fink Verlag.

Pagonis, G. (2009). Kritische Periode oder altersspezifischer Antrieb: was erklärt den Altersfaktor im Zweitspracherwerb? Eine empirische Fallstudie zum ungesteuerten Zweitspracherwerb des Deutschen durch russische Lerner unterschiedlichen Alters. Frankfurt am Main: Peter Lang GmbH. 


\section{russischsprachiger Deutschlerner}

Schmiedtová, B., Stutterheim, C. v. \& Carroll, M. (2010). Implications of language-specific patterns in event construal of advanced L2 speakers. In: A. Pavlenko Naming the world in two languages. Languages and cognition in the bilingual mind, 29-65. Bristol: Multilingual Matters.

Schmiedtová, B. \& Sahonenko, N. (2008). Die Rolle des grammatischen Aspekts in der Ereignis-Enkodierung: Ein Vergleich zwischen tschechischen und russischen Lernern des Deutschen. In: M. Walter/ P. Grommes Fortgeschrittene Lernervarietäten. Korpuslinguistik und Zweitspracherwerbsforschung, 45-72. Tübingen.

Schumacher, N. (2005) Tempus als Lerngegenstand. Ein Modell für Deutsch als Fremdsprache und seine Anwendung für italienische Lernende. Tübingen: Gunter Narr Verlag.

Schumacher, N. (2009). Perspektiven für Studien zum Gebrauch der deutschen Vergangenheitstempora durch russische L1-Sprecher. In: E. Goncharova et. al. Arbeitspapiere GIP Herzen - Humboldt. Themenheft 1: Tempus - Metaphern - Text, 28-46. St. Petersburg: Izdatel'stvo SPbGPU.

Slobin, D. (1987). Thinking for speaking. Papers from the 13th Annual Meeting of the Berkeley Linguistics Society, 435-462. Berkeley: BLS.

Slobin, D. (1996). From 'thought and language' to 'thinking for speaking'. In: von J. Gumperz / S. C. Levinson Rethinking linguistic relativity, 70-96. Cambridge: Cambridge University Press.

Smirnova, T. (2009). Aspektuale Konzepte als präferierte Muster russischer L1-Sprecher beim Lernen des Deutschen. http://www.herzen.spb.ru. Link: http://wwwnext.herzen.spb.ru/uploads/dephil-bogusl/files/Smirnova/TempusMetapher-Text-Smirnova.pdf (Zugriff am 14. August 2015).

Stutterheim, C. v. (1986). Temporalität in der Zweitsprache. Berlin: Mouton de Gruyter.

Stutterheim, C. v. (2003). Linguistic structures and information organisation. The case of very advanced learners. In: S. Foster-Cohen EUROSLA Yearbook 3, 183-206. Amsterdam: John Benjamins.

Stutterheim, C. v. \& Carroll, M. (2005). Subjektwahl und Topikkontinuität im Deutschen und Englischen. Zeitschrift für Literaturwissenschaft und Linguistik, 139, 7-27.

Stutterheim, C. v. \& Klein W. (1987). A concept-oriented approach to second language studies. In: C.W. Pfaff First and second language acquisition processes, 191 - 205. Cambridge: Newbury House.

Vendler, Z. (1967). Verbs and Times. In: Z. Vendler Linguistics in Philosophy, 97-121. Ithaca, NY: Cornell University Press.

Weinrich, H. (2001). Tempus. Besprochene und erzählte Welt. München: Beck. 